\title{
Entanglement for quantum Hall states and a generalized Chern-Simons form
}

\author{
V.P. Nairø* \\ Physics Department, City College of the CUNY, New York, New York 10031, USA
}

(Received 7 May 2020; accepted 15 June 2020; published 26 June 2020)

\begin{abstract}
We analyze some features of the entanglement entropy for an integer quantum Hall state $(\nu=1)$ in comparison with ideas from relativistic field theory and noncommutative geometry. The spectrum of the modular operator, for a restricted class of states, is shown to be similar to the case of field theory or a type $\mathrm{III}_{1}$ von Neumann algebra. We present arguments that the main part of the dependence of the entanglement entropy on background fields and geometric data such as the spin connection is given by a generalized Chern-Simons form. Implications of this result for bringing together ideas of noncommutative geometry, entropy and gravity are briefly commented upon.
\end{abstract}

DOI: $10.1103 /$ PhysRevD.101.125021

\section{INTRODUCTION}

The idea that there is some deep connection between entropy and gravity is by now well-known and wellaccepted [1-4]. Also the Reeh-Schlieder and ConnesStormer theorems [5], coupled with the observation that the algebra of local observables should be a type $\mathrm{III}_{1}$ von Neumann algebra, tell us that entanglement is an integral part of relativistic quantum field theory [6-8]. In noncommutative geometry, one attributes degrees of freedom to space itself via its description by the states of a suitable Hilbert space [9]. Putting these three observations together, a question which naturally arises is whether we can calculate an entanglement entropy between states defining the spatial geometry in the noncommutative scenario and relate it to gravity. This is the subject we explore in this paper.

A simple working model for noncommutative geometry is given by the quantum Hall system. If we consider a Kähler manifold $\mathcal{M}$, we can choose a background magnetic field which is proportional to the Kähler two-form. The lowest Landau level (LLL) is obtained by quantizing $\mathcal{M}$ with this multiple of the Kähler form as the symplectic structure. We get a Hilbert space $\mathcal{H}$ which is spanned by holomorphic wave functions and which can be used as the model for the noncommutative version of $\mathcal{M}[10,11]$. Although we phrased this in terms of a Landau-Hall problem, the states of the LLL can be viewed as

\footnotetext{
*vpnair@ccny.cuny.edu
}

Published by the American Physical Society under the terms of the Creative Commons Attribution 4.0 International license. Further distribution of this work must maintain attribution to the author(s) and the published article's title, journal citation, and DOI. Funded by SCOAP ${ }^{3}$. holomorphic sections of a power of the canonical line bundle, so the tie-in to the physical situation of the Hall effect is useful but not essential. On the other hand, what we do in this paper can also be viewed more narrowly as an interesting view on some entanglement issues for the Hall system, ignoring the larger perspective of gravity and noncommutative geometry.

We consider a completely filled LLL and a surface (of co-dimension 1) separating $\mathcal{M}$ into two regions. It is then possible to define an algebra of local observables and reduced density matrices. Regarding the entanglement between the states in the two regions, we consider the spectrum of the modular operator and show that it is basically $\mathbb{R}_{+}$. If we consider only the fully filled LLL, which is what is relevant in modeling noncommutative geometry, the only freedom in the reduced density matrices is due to a change of the separating surface in $\mathcal{M}$. For all such cases, we will see that the spectrum of the modular operator is $\mathbb{R}_{+}$, as the number of states tends to infinity. In relativistic quantum field theory, the algebra of local observables is expected to be a type $\mathrm{III}_{1}$ von Neumann algebra. This means that the intersection of the spectra of the modular operators over all states (or density matrices) should be $\mathbb{R}_{+}[6,7]$. In the present problem, we do not exactly have this result as we are not considering all possible density matrices. For the fully filled LLL, as mentioned above, only a smaller class of density matrices is meaningful. Over this set of reduced density matrices, we do obtain the same spectrum, namely, $\mathbb{R}_{+}$.

The second part of our analysis focuses on the changes in the entanglement entropy as the background fields are varied. We can consider fluctuations in the magnetic field as well as changes in the background geometry due to gravitational fluctuations. We show that the entanglement entropy, as a function of these background fields, is 
proportional to a Chern-Simons action. It should be emphasized that we are not discussing the effective action for the Hall states; it would not be deemed surprising that the latter is a Chern-Simons theory. To highlight the nature of our result, consider the fact that Einstein gravity in $2+1$ dimensions is described by a Chern-Simons theory [12]. There have also been investigations of higher dimensional Chern-Simons gravities recently [13]. In these theories, the gravitational field equations are the extremization of the CS action. Our argument shows that they may be related to the extremization of the entanglement entropy between states corresponding to the degrees of freedom of space itself.

In Sec. II, we recall a few relevant results from relativistic quantum field theory. The spectrum of the modular operator for Landau-Hall states on $S^{2}$ (or fuzzy version of $S^{2}$ ) is considered in Sec. III. The generalization to $\mathbb{C P}^{k}$, $k>1$, is done in Sec. IV. The background field dependence of the entanglement entropy is discussed in Sec. V. The paper concludes with a short discussion and two Appendixes with explicit details of some of the relevant calculations.

\section{OBSERVATIONS FROM FIELD THEORY}

In this section, we collect a few known observations about relativistic quantum field theory which can serve as points of comparison for our analysis for Hall states.

A key property of relativistic quantum field theory (QFT) is that local observables commute at spacelike separations,

$$
[\phi(x), \phi(y)]=0, \quad(x-y)^{2}<0
$$

Here $\phi(x)$ are not necessarily fundamental or elementary fields. In some neighborhood $\mathcal{O}$ of spacetime, we can define a local algebra of observables, denoted by $\mathcal{A}(\mathcal{O})$, defined by bounded operators of the form

$$
\phi(f)=\int f(x) \phi(x)
$$

where the support of $f(x)$ is contained in $\mathcal{O} \cdot \mathcal{A}(\mathcal{O})$ forms a subalgebra of $\mathcal{B}(\mathcal{H})$, the set of bounded operators on the Hilbert space $\mathcal{H}$. It is unital in the sense that it includes the identity and is a $*$-algebra since it inherits an involution corresponding to the adjoint operation. If $\mathcal{O}^{\prime}$ is the causal complement of $\mathcal{O}$, then (1) translates as

$$
\left[\mathcal{A}(\mathcal{O}), \mathcal{A}\left(\mathcal{O}^{\prime}\right)\right]=0
$$

This tells us that $\mathcal{A}\left(\mathcal{O}^{\prime}\right)$ is contained in the commutant $\mathcal{A}^{\prime}$ of $\mathcal{A}(\mathcal{O})$, namely the set of all operators which commute with $\mathcal{A}(\mathcal{O})$. Following Haag, we take $\mathcal{A}\left(\mathcal{O}^{\prime}\right)=\mathcal{A}(\mathcal{O})^{\prime}$, a statement which is known as Haag duality. We also assume that $\mathcal{A}(\mathcal{O})=\mathcal{A}(O)^{\prime \prime}$. A unital $*$-subalgebra $\mathcal{A}$ of $\mathcal{B}(\mathcal{H})$ which has the property $\mathcal{A}=\mathcal{A}^{\prime \prime}$ is a von Neumann algebra. (There are other definitions based on operator topology, but this is the simplest for our purpose.) Thus, we can treat $\mathcal{A}(\mathcal{O})$ and $\mathcal{A}\left(\mathcal{O}^{\prime}\right)\left(=\mathcal{A}(\mathcal{O})^{\prime}\right)$ as von Neumann algebras. In what follows, we will consider fields at a given time. Strictly speaking, the definition of local operators will need pointsplitting in time, but this refinement will not be important for most of the following discussion.

While one can define an algebra of local observables, the Hilbert space of states does not factorize into Hilbert subspaces defined locally. This statement is the result of some deep theorems, but a simple illustrative example which highlights this feature is obtained in terms of local single-particle states. Consider defining "local" one-particle states of the form

$|f\rangle=\int d^{3} x f(x) \psi^{\dagger}(x)|0\rangle, \quad|h\rangle=\int d^{3} x h(x) \psi^{\dagger}(x)|0\rangle$

where the functions $f(x)$ and $h(x)$ have supports in disjoint regions of space and $\psi^{\dagger}$ denotes the negative frequency (creation) part of an elementary field operator, which may be taken, for the present purpose, as a free field for simplicity; we may denote this field as $\varphi=\psi+\psi^{\dagger}$. The overlap of the states (4) is given by

$$
\langle f \mid h\rangle=\int d^{3} x d^{3} y \frac{d^{3} k}{(2 \pi)^{3}} \frac{1}{2 \omega_{k}} e^{-i \vec{k} \cdot(\vec{x}-\vec{y})} f(x) h(y)
$$

where $\omega_{k}=\sqrt{k^{2}+m^{2}}$. (We consider particles of mass $m$.) The factor $1 /\left(2 \omega_{k}\right)$, which is characteristic of the relativistic theory, plays a crucial role. In the nonrelativistic case where $\omega_{k} \approx m$, this factor is a constant, independent of $k$, and the integration over $k$ gives a $\delta$-function and hence the overlap integral is zero since $f$ and $h$ have no overlap for their supports. But in the relativistic theory, we see that this overlap is nonzero, rendering void any attempt to define local one-particle states. Entanglement thus becomes a characteristic feature of relativistic field theory.

A different but related way to see this is the following. Notice that the correlation function involved in (5) can be obtained from the vacuum wave function for the scalar field theory

$$
\Psi_{0}=\mathcal{N} \exp \left(-\frac{1}{2} \int_{x, y} \varphi(x)\left(\omega_{k}\right)_{x, y} \varphi(y)\right)
$$

The appearance of $\omega_{k}$ can be traced to the Lorentz invariance of the vacuum state. The key point for us is that the operator $\left(\omega_{k}\right)_{x, y}$ is nonlocal. Thus if we consider field configurations $\varphi_{1}$ and $\varphi_{2}$ with support in disjoint regions, there is still nontrivial overlap in the wave function since $\int \varphi_{1} \omega_{k} \varphi_{2} \neq 0$. This prevents the factorization of the vacuum wave function, and all other states, the latter being 
obtained form the vacuum by the action of various creation operators. ${ }^{1}$

Another important feature is embodied in the ReehSchlieder theorem which tells us that the local algebra $\mathcal{A}(\mathcal{O})$ is sufficient to generate a dense set of states on the Hilbert space of the theory by their action, say, on the vacuum state [8]. In other words, the vacuum state $|0\rangle$ is a cyclic vector for the algebra $\mathcal{A}(\mathcal{O})$. For such a state, if $A^{\prime}|0\rangle=0$ for $A^{\prime} \in \mathcal{A}^{\prime}$, then

$$
0=A A^{\prime}|0\rangle=A^{\prime} A|0\rangle
$$

where we use the fact that $A$ and $A^{\prime}$ commute. Since $A|0\rangle$ generates a dense set of states, considering all $A \in \mathcal{A}$, we see that $A^{\prime}$ should vanish on a dense set of states, hence $A^{\prime}=0$. A vector $|\Psi\rangle$ is said to be a separating vector if $A^{\prime}|\Psi\rangle=0$ implies $A^{\prime}=0$. Thus a cyclic vector for $\mathcal{A}$ is separating for $\mathcal{A}^{\prime}$ and vice versa. Similar arguments apply starting from $\mathcal{A}^{\prime}$, so we have the result that the vacuum $|0\rangle$ is cyclic and separating for both $\mathcal{A}(\mathcal{O})$ and $\mathcal{A}(\mathcal{O})^{\prime}{ }^{2}$ This is the required premise for the Tomita-Takesaki theorem. Toward the statement of the theorem, let $|\Psi\rangle$ be a cyclic and separating vector for the von Neumann algebra $\mathcal{A}$. (We will use a general state $|\Psi\rangle$ for many of the statements here, although specializing to the vacuum state $|0\rangle$ is most pertinent to the general field theory analysis.) We can then define an antilinear map $S_{\Psi}$ whose action is given by

$$
S_{\Psi}(A|\Psi\rangle)=A^{\dagger}|\Psi\rangle
$$

Evidently $S_{\Psi}^{2}=1$. Since $S_{\Psi}$ is antilinear, conjugation is defined by (see [8]),

$$
\langle\alpha \mid S \beta\rangle=\overline{\left\langle S^{\dagger} \alpha \mid \beta\right\rangle}=\left\langle\beta \mid S^{\dagger} \alpha\right\rangle
$$

Thus if we define $F A^{\prime}|\Psi\rangle=A^{\prime \dagger}|\Psi\rangle$,

$$
\begin{aligned}
\left\langle A^{\prime} \Psi \mid S A \Psi\right\rangle & =\left\langle A^{\prime} \Psi \mid A^{\dagger} \Psi\right\rangle=\left\langle A A^{\prime} \Psi \mid \Psi\right\rangle \\
& =\left\langle A^{\prime} A \Psi \mid \Psi\right\rangle=\left\langle A \Psi \mid A^{\prime \dagger} \Psi\right\rangle=\left\langle A \Psi \mid F A^{\prime} \Psi\right\rangle
\end{aligned}
$$

Taking $|\alpha\rangle=\left|A^{\prime} \Psi\right\rangle,|\beta\rangle=|A \Psi\rangle$, we see from (9) that $F=S^{\dagger}$. Thus $S^{\dagger}$ acts on $\mathcal{A}^{\prime}$ as $S$ acts on $\mathcal{A}$. Going back to (8), the states $A|\Psi\rangle$ and $A^{\dagger}|\Psi\rangle$ do not have the same norm in general, so $S_{\Psi}$ is not unimodular. We can separate out a

\footnotetext{
${ }^{1}$ There are many physical systems and Hamiltonians, e.g., a spin chain, which lead to long correlations but allow factorization of the Hilbert space. There is no contradiction. All separations in a nonrelativistic system are timelike, since the speed of light can be taken as infinite. Also, Lorentz invariance is crucial to the statement of nonfactorizability. Discrete systems do not fulfill this premise.

${ }^{2} \mathrm{~A}$ related statement or corollary is that there is no bounded local operator which annihilates the vacuum state, a statement which is useful for proving Coleman's theorem on realizations of symmetry.
}

unimodular part $J$ via the polar decomposition $S=J \Delta^{\frac{1}{2}}$. where $J$ is a unimodular antilinear operator and $\Delta$ is selfadjoint. $J$ is referred to as the modular conjugation and $\Delta$ is the Tomita modular operator. The latter can also be defined by

$$
\Delta_{\Psi}=S_{\Psi}^{\dagger} S_{\Psi}
$$

This operator depends on the choice of the state $|\Psi\rangle$. Among other useful properties of $S, S^{\dagger}$, we can easily verify that $J^{\dagger}=J$ and

$$
\begin{array}{ll}
J^{2}=J^{\dagger} J=1, & J \Delta^{\frac{1}{2}} J=\Delta^{-\frac{1}{2}} \\
S_{\Psi}^{\dagger}=\Delta_{\Psi}^{\frac{1}{2}} J^{\dagger}, & S_{\Psi} S_{\Psi}^{\dagger}=\Delta_{\Psi}^{-1}
\end{array}
$$

The Tomita-Takesaki theorem is the statement that given a von Neumann algebra $\mathcal{A}$ and a cyclic and separating vector $|\Psi\rangle$, with $S$ and $S^{\dagger}$ as defined above,

$$
\begin{aligned}
J \mathcal{A} J & =\mathcal{A}^{\prime} \\
\Delta^{i t} \mathcal{A} \Delta^{-i t} & =\mathcal{A}, \quad \text { for all } t \in \mathbb{R}
\end{aligned}
$$

The first statement relates $\mathcal{A}$ and $\mathcal{A}^{\prime}$, while the second identifies a one-parameter family of automorphisms of $\mathcal{A}$ which may be viewed as time-evolution. The proof of the theorem is very involved, we do not discuss it, but to see how such statements could arise, notice that, if $A, B \in \mathcal{A}$,

$$
\begin{aligned}
& S B S A|\Psi\rangle=S B A^{\dagger}|\Psi\rangle=A B^{\dagger}|\Psi\rangle \\
& A S B S|\Psi\rangle=A S B|\Psi\rangle=A B^{\dagger}|\Psi\rangle
\end{aligned}
$$

Thus $S B S$ is contained in $\mathcal{A}^{\prime}$. Reducing this to the unimodular part of the action of $S$, we see how the first part of the theorem could arise.

Using the polar decomposition, (8) gives $J \Delta^{\frac{1}{2}} A|\Psi\rangle=$ $A^{\dagger}|\Psi\rangle$. Thus $\Delta^{\frac{1}{2}} A|\Psi\rangle$ and $A^{\dagger}|\Psi\rangle$ should have the same norm. Taking $|\Psi\rangle$ to be the vacuum $|0\rangle$, since $A$ is in the algebra of local observables in $\mathcal{O}$, we can define the unimodularity condition for $J$ as

$$
\operatorname{Tr}\left(\rho_{\mathcal{O}} A^{\dagger} \Delta A\right)=\operatorname{Tr}\left(\rho_{\mathcal{O}} A A^{\dagger}\right)
$$

where $\rho_{\mathcal{O}}$ is the reduced density matrix starting from $|0\rangle$. (We may view the vacuum in terms of its wave function as a functional of the fields and we can integrate out the part of the fields corresponding to $\mathcal{O}^{\prime}$ to obtain this. How exactly this is done is not important for now.) There is a similar equality for operators in $\mathcal{A}^{\prime}$ with a reduced density matrix $\rho_{\mathcal{O}^{\prime}}^{\prime}$. Notice that the relation (15) and the corresponding one for operators in $\mathcal{A}^{\prime}$ are obtained if we define the action of $\Delta$ by 


$$
\begin{aligned}
\Delta A & =\rho_{\mathcal{O}} \rho_{\mathcal{O}^{\prime}}^{\prime-1} A \rho_{\mathcal{O}^{\prime}}^{\prime} \rho_{\mathcal{O}}^{-1} \\
\Delta A^{\prime} & =\rho_{\mathcal{O}} \rho_{\mathcal{O}^{\prime}}^{\prime-1} A^{\prime} \rho_{\mathcal{O}^{\prime}}^{\prime} \rho_{\mathcal{O}}^{-1}
\end{aligned}
$$

Since the vacuum state is a separating vector, $\rho_{\mathcal{O}}^{-1}$ and $\rho_{\mathcal{O}^{\prime}}^{-1}$ do exist and these formulas are well defined. Introducing states $|k, \tilde{k}\rangle$ which form a basis for $\mathcal{H} \otimes \mathcal{H}$, we can represent the operators $A$ and $A^{\prime}$ in the form ${ }^{3}$

$$
A=\sum_{k, \tilde{k}}|k, \tilde{k}\rangle\left(A_{k l} \delta_{\tilde{k} \tilde{l}}\right)\left\langle l, \tilde{l}\left|, \quad A^{\prime}=\sum_{k, \tilde{k}}\right| k, \tilde{k}\right\rangle\left(\delta_{k l} A_{\tilde{k} \tilde{l}}^{\prime}\right)\langle l, \tilde{l}|
$$

The action of $\Delta$ as in (16) can then be written as

$$
\Delta|k, \tilde{k}\rangle=\sum_{l, \tilde{l}}\left(\rho_{\mathcal{O}}\right)_{k l}\left(\rho_{\mathcal{O}^{\prime}}^{\prime-1}\right)_{\tilde{k} \tilde{l}}|l, \tilde{l}\rangle
$$

A more convenient notation is to represent $|k, \tilde{k}\rangle$ as $\Phi=|k\rangle\langle\tilde{k}|$, so that the action of $\Delta$ can be represented as [8]

$$
\begin{aligned}
\Delta_{\Psi} \Phi & =\sum_{l, \tilde{l}}\left(\rho_{\mathcal{O}}\right)_{k l}\left(\rho_{\mathcal{O}^{\prime}}^{\prime-1}\right)_{\tilde{k} \tilde{l}}|k\rangle\left\langle\tilde{k}\left|=\sum_{l, \tilde{l}}\left(\rho_{\mathcal{O}}\right)_{k l}\right| k\right\rangle\langle\tilde{k}|\left[\left(\rho_{\mathcal{O}^{\prime}}^{\prime T}\right)^{-1}\right]_{\tilde{l} \tilde{k}} \\
& =\rho_{\mathcal{O}} \Phi\left(\rho_{\mathcal{O}^{\prime}}^{\prime T}\right)^{-1}
\end{aligned}
$$

A similar equation holds for a more general state $\Phi=\sum c_{k, \tilde{k}}|k, \tilde{k}\rangle=\sum c_{k, \tilde{k}}|k\rangle\langle\tilde{k}|$. It should be emphasized that the dependence of $\Delta_{\Psi}$ on the state $|\Psi\rangle=|0\rangle$ is carried by the reduced density matrices, while $\Phi$ denotes another possible state for the algebras.

From the point of view of general analyses of von Neumann algebras, the importance of the modular operator $\Delta$ is that its spectrum can be used to classify such algebras $[6,7]$. The von Neumann algebra of local operators in relativistic quantum field theory is expected to be of the hyperfinite Type $\mathrm{III}_{1}$. This is characterized by the property that

$$
\bigcap_{\Psi} \operatorname{Spec}\left(\Delta_{\Psi}\right)=\mathbb{R}_{+}
$$

In other words, the intersection of the spectra over all choices of the state $|\Psi\rangle$ is $\mathbb{R}_{+}$. The spectrum itself is defined by the action of $\Delta_{\Psi}$ on a general state $\Phi$ as in (19). A consequence of the algebra being of the hyperfinite Type $\mathrm{III}_{1}$ is that any state can be brought arbitrarily close to any other state by unitary transformations defined separately in $\mathcal{A}(\mathcal{O})$ and $\mathcal{A}\left(\mathcal{O}^{\prime}\right)$. This is the essence of the ConnesStormer theorem and implies that almost all states are entangled $[6,7]$.

\footnotetext{
${ }^{3} \mathrm{We}$ use discrete labels and summation signs to give the general tenor of the results and to write expressions in a form suitable for later sections. An appropriate limit will be needed for the continuum field theory.
}

In the light of these facts about relativistic quantum field theory, we first consider the natural question of whether, for a quantum Hall state on a manifold divided into two regions, the spectrum of the modular operator is $\mathbb{R}_{+}$. We show that this is indeed the case in a limited sense. This is discussed in the next two sections. We shall then take up the question of how the entanglement entropy depends on the background gauge fields and the spin connection.

\section{THE SPECTRUM OF THE MODULAR OPERATOR FOR THE $\nu=1$ HALL STATE}

We start by considering the quantum Hall state on the two-sphere $S^{2}$ where the lowest Landau level is fully occupied, i.e., the $\nu=1$ state [14]. The fermion field operators can be expanded as

$$
\psi=\sum_{s} a_{s} u_{s}(x)+\sum_{\alpha} a_{\alpha} U_{\alpha}(x)
$$

where $u_{s}(x)$ are the single particle wave functions for the lowest Landau level (LLL). $U_{\alpha}$ denote the higher Landau level wave functions, which will not be very important for what follows. The fully occupied state LLL can thus be specified as

$$
|\nu=1\rangle=a_{0}^{\dagger} a_{1}^{\dagger} \cdots a_{n}^{\dagger}|0\rangle
$$

where $n+1$ denotes the number of states which constitute the LLL. For $S^{2}, n=2 B r^{2}$ where $B$ is the radial magnetic field of a monopole at the origin in the standard embedding of $S^{2}$ in $\mathbb{R}^{3}$. From (21), the annihilation and creation operators for the LLL may be expressed as

$$
a_{s}=\int d \mu u_{s}^{*} \psi, \quad a_{s}^{\dagger}=\int d \mu u_{s} \psi^{\dagger}
$$

We can parametrize the sphere in terms of complex coordinates $z, \bar{z}$, corresponding to the stereographic projection of $S^{2}$ onto the plane. The wave functions are then given by

$$
u_{s}(x)=\frac{1}{\sqrt{\pi}} \sqrt{\frac{\Gamma(n+2)}{s ! \Gamma(n-s+1)}} \frac{z^{s}}{(1+\bar{z} z)^{n / 2}} .
$$

We want to separate the sphere into two regions, say, the northern hemisphere and the southern hemisphere. The equator, which is the dividing line, corresponds to $|z|=1$ in the coordinates we are using. We thus define

$$
\begin{array}{ll}
b_{s}=\frac{1}{\sqrt{\lambda_{s}}} \int_{0}^{|z|=1} d \mu u_{s}^{*} \psi, & b_{s}^{\dagger}=\frac{1}{\sqrt{\lambda_{s}}} \int_{0}^{|z|=1} d \mu u_{s} \psi^{\dagger} \\
c_{s}=\frac{1}{\sqrt{1-\lambda_{s}}} \int_{|z|=1}^{\infty} d \mu u_{s}^{*} \psi, & c_{s}^{\dagger}=\frac{1}{\sqrt{1-\lambda_{s}}} \int_{|z|=1}^{\infty} d \mu u_{s} \psi^{\dagger}
\end{array}
$$


where

$$
\lambda_{s}=\int_{0}^{|z|=1} u_{s}^{*} u_{s}
$$

In terms of these operators

$$
\begin{aligned}
& a_{s}=\sqrt{\lambda_{s}} b_{s}+\sqrt{1-\lambda_{s}} c_{s} \\
& a_{s}^{\dagger}=\sqrt{\lambda_{s}} b_{s}^{\dagger}+\sqrt{1-\lambda_{s}} c_{s}^{\dagger} .
\end{aligned}
$$

The operators $\left\{b_{s}, b_{s}^{\dagger}\right\}$ and $\left\{c_{s}, c_{s}^{\dagger}\right\}$ form two mutually commuting fermion algebras, obeying

$$
\begin{aligned}
& \left\{b_{s}, b_{r}\right\}=\left\{b_{s}^{\dagger}, b_{r}^{\dagger}\right\}=0=\left\{c_{s}, c_{r}\right\}=\left\{c_{s}^{\dagger}, c_{r}^{\dagger}\right\} \\
& \left\{b_{s}, b_{r}^{\dagger}\right\}=\delta_{r s}=\left\{c_{s}, c_{r}^{\dagger}\right\} .
\end{aligned}
$$

The second set of commutation rules requires the definition of the normalization factor of $\sqrt{\lambda_{s}}, \sqrt{1-\lambda_{s}}$ in (25). In verifying (28), we also assume that the angular integrations suffice to make the integral vanish for $r \neq s$. This is indeed the case and will be important for the higher dimensional generalization.

A short parenthetical remark may be useful before we go on. If we consider functions which have support only in the region $|z|<1$, the lowest Landau level wave functions $\left\{u_{s}\right\}$ are not an adequate basis for a mode expansion of such functions. One can get a complete basis by including the higher Landau levels as well. This is also clear from using the full mode expansion (21) for $\psi$ and $\psi^{\dagger}$ in (25). We then see that the operator expressions for $b_{s}, b_{s}^{\dagger}$ and $c_{s}, c_{s}^{\dagger}$ will also involve $a_{\alpha}, a_{\alpha}^{\dagger}$ where the subscript $\alpha$ refers to the higher LLs.

Returning to the main chain of reasoning, a state vector for one fermion occupying the state corresponding to $u_{s}$ is given by

$$
|s\rangle=a_{s}^{\dagger}|0\rangle=\sqrt{\lambda_{s}} b_{s}^{\dagger}|0\rangle+\sqrt{1-\lambda_{s}} c_{s}^{\dagger}|0\rangle .
$$

This defines a way of splitting the state in terms of degrees of freedom corresponding to the inside region $|z|<1$ and the outside region $|z|>1$. In fact, we can consider local observables which correspond to independent unitary transformations of the $b_{s}$ 's and the $c_{s}$ 's. Thus let $\mathcal{A}$ denote the set of all unitary transformations $U_{s r}$ on $b_{s}$ (and $b_{s}^{\dagger}$ ), of the form $b_{s} \rightarrow U_{s r} b_{r}$, and $\tilde{\mathcal{A}}$ denote the set of unitary transformations $V_{s r}$ of the form $c_{s} \rightarrow V_{s r} c_{r}$. These may be interpreted as the algebra of observables for the region inside (i.e., $\mathcal{A}$ ) and the region outside (i.e., $\mathcal{A}^{\prime}=\tilde{\mathcal{A}}$ ), respectively. Evidently, these form two mutually commuting algebras which are copies of $U(n+1)$,

$$
[\mathcal{A}, \tilde{\mathcal{A}}]=0 .
$$

The state (29) is, of course, entangled, since $\lambda_{s} \neq 0,1$ in general. Since the only operator which commutes with all of $U_{s r}$ is the identity and similarly for $V_{s r}$, we see that $\mathcal{A}$ is the commutant of $\tilde{\mathcal{A}}$ and vice versa. Thus we have two von Neumann algebras, which become infinite dimensional as we take $n \rightarrow \infty$. The state corresponding to (22), expressed in terms of the algebra as a density matrix, can be written, upon using (29), as

$$
\begin{aligned}
\rho= & \prod_{\otimes_{s}}\left[\lambda_{s} b_{s}^{\dagger}|0\rangle\left\langle 0\left|b_{s}+\left(1-\lambda_{s}\right) c_{s}^{\dagger}\right| 0\right\rangle\langle 0| c_{s}\right. \\
& \left.+\sqrt{\lambda_{s}\left(1-\lambda_{s}\right)}\left(b_{s}^{\dagger}|0\rangle\left\langle 0\left|c_{s}+c_{s}^{\dagger}\right| 0\right\rangle\langle 0| b_{s}\right)\right] .
\end{aligned}
$$

Notice that the state $c_{s}^{\dagger}|0\rangle$ has "one particle of the $c$-type" although the occupation number for the $b$-type is zero and vice versa. We can now trace over the $c$-states to get a reduced density matrix for the $b$-type, and similarly for the $c$-type. These are given by

$$
\begin{aligned}
& \rho_{b}=\prod_{\otimes_{s}}\left[\lambda_{s} b_{s}^{\dagger}|0\rangle\left\langle 0\left|b_{s}+\left(1-\lambda_{s}\right)\right| 0\right\rangle\langle 0|\right] \equiv \prod_{\bigotimes_{s}}\left(\rho_{b}\right)_{s} \\
& \rho_{c}=\prod_{\bigotimes_{s}}\left[\left(1-\lambda_{s}\right) c_{s}^{\dagger}|0\rangle\left\langle 0\left|c_{s}+\lambda_{s}\right| 0\right\rangle\langle 0|\right] \equiv \prod_{\bigotimes_{s}}\left(\rho_{c}\right)_{s} .
\end{aligned}
$$

There is a slight abuse of notation here in continuing to use $|0\rangle$. It should be noted that, in $\rho_{b}$, while the state $|0\rangle\langle 0|$, which is obtained by tracing over the $c$ 's, has no $b$-occupancy, it is not empty. It stands for $\sum_{s} c_{s}^{\dagger}|0, \tilde{0}\rangle\langle 0, \tilde{0}| c_{s}$ if we consider a more elaborate notation of two copies of $\mathcal{H}$ as in Sec. II. Thus $|0\rangle\langle 0|$ in the first line of (32) does capture the effect of fermions outside $|z|=1$, although the effect is small, since $1-\lambda_{s}$ will be small for states localized far into the outside region, i.e., for states with $s \gg \frac{1}{2} n$. And a similar statement, mutatis mutandis, holds for the second line of (32) as well. In each of the cases in (32) one can define the von Neumann entropy, which is also the entanglement entropy,

$$
\begin{aligned}
S & =-\operatorname{Tr}\left(\rho_{b} \log \rho_{b}\right)=-\operatorname{Tr}\left(\rho_{c} \log \rho_{c}\right) \\
& =-\sum_{s}\left[\lambda_{s} \log \lambda_{s}+\left(1-\lambda_{s}\right) \log \left(1-\lambda_{s}\right)\right]
\end{aligned}
$$

This method of splitting $a_{s}, a_{s}^{\dagger}$ as in (27) and calculating the entanglement entropy was first given in [15]. For entanglement entropy for the $\nu=1$ state in two dimensions, and in higher dimensions, for a fixed background, see also [16]. Some of the other references on the entanglement entropy for Hall systems are given in $[17,18]$.

We now want to consider the modular operator corresponding to the states $\rho_{b}, \rho_{c}$. A general state can be taken to be of the form 


$$
\Phi=\prod_{\otimes_{s}}\left[\begin{array}{cc}
\alpha+\beta & \gamma-i \delta \\
\gamma+i \delta & \alpha-\beta
\end{array}\right]_{s}
$$

with arbitrary elements $\alpha, \ldots, \delta$. In this notation, the (11) element corresponds to $b^{\dagger}|0\rangle\langle 0| b$, (12) to $b^{\dagger}|0\rangle\langle 0| c$, (21) to $c^{\dagger}|0\rangle\langle 0| b$, (22) to $c^{\dagger}|0\rangle\langle 0| c$. Following [8] and our discussion in Sec. II, we can define the action of the modular operator $\Delta$ as

$$
\Delta \Phi=\rho_{b} \Phi\left(\rho_{c}\right)^{-1} .
$$

Just to reiterate, in this expression, the state dependence of $\Delta$ is given in terms of $\rho_{b}, \rho_{c}$. For each $2 \times 2$ subspace, (35) works out to

$$
\Delta\left(\begin{array}{l}
\alpha \\
\beta \\
\gamma \\
\delta
\end{array}\right)=\left[\begin{array}{cccc}
1 & 0 & 0 & 0 \\
0 & 1 & 0 & 0 \\
0 & 0 & \frac{1}{2}\left(x+x^{-1}\right) & -\frac{i}{2}\left(x-x^{-1}\right) \\
0 & 0 & \frac{i}{2}\left(x-x^{-1}\right) & \frac{1}{2}\left(x+x^{-1}\right)
\end{array}\right]\left(\begin{array}{l}
\alpha \\
\beta \\
\gamma \\
\delta
\end{array}\right)
$$

where $x=\lambda /(1-\lambda)$. The eigenvalues are $1,1, \lambda /(1-\lambda)$, $(1-\lambda) / \lambda$. This is for one value of $s$. The full spectrum is thus given by the product of these eigenvalues over all values of $s$. Thus

$$
\operatorname{Spec}(\Delta)=\left(\{1\},\{1\},\left\{\lambda_{s} /\left(1-\lambda_{s}\right)\right\},\left\{\left(1-\lambda_{s}\right) / \lambda_{s}\right\},\left\{\lambda_{s_{1}} \lambda_{s_{2}} /\left(1-\lambda_{s_{1}}\right) /\left(1-\lambda_{s_{2}}\right)\right\}, \text { etc. }\right) .
$$

The products of the individual eigenvalues for all values of $s$ are included in this set. Since some of the eigenvalues are just 1 , the individual eigenvalues get repeated as well in this set.

Our first result is to show that, as $n \rightarrow \infty$ for states on $S^{2}$, for any value between zero and 1 , there is some $k$ such that $\lambda_{s}$ is equal to this chosen value. This will imply that the spectrum of $\Delta$ is the interval $[0, \infty)$. The calculations are given in Appendix A. We show that the values of $\lambda_{s}$ start near 1 for $s \ll n$ and drop to zero as $s$ becomes close to $n$. The maximal difference of $\lambda_{s}$ for nearby values of $s$ occurs at $s=\frac{1}{2} n$, where

$$
\frac{\lambda_{\frac{n}{2}}-\lambda_{\frac{n}{2}+1}}{\lambda_{\frac{n}{2}}}=\sqrt{\frac{8}{\pi}} \frac{1}{\sqrt{n}}+\mathcal{O}(1 / n)
$$

We can therefore conclude that the differences between $\lambda_{s}$ and $\lambda_{s+1}$ vanish for all $s$, as $n \rightarrow \infty$, showing that the values of $\lambda_{s}$ fill the interval between zero and 1 . In other words, the spectrum of $\Delta$ for this state is $\mathbb{R}_{+}$.

For the analysis given above, we chose the dividing line between the two regions as the equator, at $|z|=1$. The result can be generalized to an arbitrary value of $|z|$ for the dividing line, so long as the number of states in each region tends to infinity as $n \rightarrow \infty$. Consider $|z|=R$. The relevant integral is now

$$
\lambda_{s}=\frac{(n+1) !}{s !(n-s) !} \int_{0}^{R^{2}} d u \frac{u^{s}}{(1+u)^{n+2}} .
$$

The middle of the transition region between $\lambda=0$ and $\lambda=1$ will occur at $s=s_{*}=R^{2} n /\left(1+R^{2}\right)$. In this case, the maximal difference is given by

$$
\frac{\lambda_{s_{*}}-\lambda_{s_{*}+1}}{\lambda_{s_{*}}} \approx \sqrt{\frac{2}{w(1-w) \pi}} \frac{1}{\sqrt{n}}+\cdots, \quad w=\frac{R^{2}}{1+R^{2}} .
$$

Once again, we notice that the differences of the nearby $\lambda$ 's vanish as $n \rightarrow \infty$. Thus the values of $\lambda$ will fill the interval between zero and 1 , leading to the spectrum of $\Delta$ as $\mathbb{R}_{+}$.

Deformations of the dividing line can be viewed as area preserving diffeomorphims which are realized in terms of unitary transformations of the lowest Landau levels states. The spectrum of $\Delta$ will not be sensitive to this, so the conclusion holds more generally than for the case of a circular dividing line.

If we consider the lowest Landau level as a model for the fuzzy version of $S^{2}$, then the relevant state must be the fully filled level with $\nu=1$. Having unfilled one-particle states will correspond to having the two-sphere with points removed, as $n \rightarrow \infty$. Therefore the only set of states relevant for the case of fuzzy $S^{2}$ will correspond to different choices of $R$. This leads to the conclusion:

For all allowable states in the framework of using the lowest Landau as a model for a noncommutative space, $\bigcap_{\Psi} \operatorname{Spec}\left(\Delta_{\Psi}\right)=\mathbb{R}_{+}$.

\section{GENERALIZATION TO $\mathbb{C P}^{k}$}

The results we have obtained for $S^{2} \sim \mathbb{C P}^{1}$ can be easily generalized to $\mathbb{C P}^{k}, k>1[11]$. We may view this space as a group coset,

$$
\mathbb{C P}^{k}=\frac{S U(k+1)}{U(k)}
$$

This is a homogeneous space with $U(k)$ as the isotropy group. The curvature is thus valued in the Lie algebra of 
$U(k)$ and is constant in the tangent frame basis. This means that we can introduce additional gauge fields with the field strength proportional to the curvatures and thus set up the analogue of the Landau problem and Hall effect. More explicitly, the wave functions can be considered as functions on $S U(k+1)$ which have a specific transformation property under the $U(k) \subset S U(k+1)$. A basis for functions on the group $S U(k+1)$ is given by the matrices corresponding to the group elements in the unitary irreducible representations, or the so-called Wigner $\mathcal{D}$-functions, which are defined as

$$
\mathcal{D}_{\mathfrak{l} ; \mathfrak{r}}^{(J)}(g)=\langle J, \mathfrak{l}|g| J, \mathfrak{r}\rangle
$$

where $J$ denotes the irreducible representation and $\mathfrak{l}, \mathfrak{r}$ stand for two sets of quantum numbers specifying the states within the representation. There is a natural left and right action of group translations on an element $g \in S U(k+1)$, defined by

$$
\hat{L}_{A} g=T_{A} g, \quad \hat{R}_{A} g=g T_{A}
$$

where $T_{A}$ are the $S U(k+1)$ generators in the representation to which $g$ belongs.

The generators of $S U(k+1)$ which are not in the algebra of $U(k) \subset S U(k+1)$ can be separated into $T_{+i}$, $i=1,2, \ldots, k$, which are of the raising type and $T_{-i}$ which are of the lowering type. These generate translations while $U(k)$ generates rotations at a point. The covariant derivatives on $\mathbb{C P}^{k}$ are given by

$$
\mathcal{D}_{ \pm i}=i \frac{\hat{R}_{ \pm i}}{r}
$$

where $r$ is a parameter with the dimensions of length. (The volume of the manifold will be proportional to $r^{2 k}$.) The strength of the gauge field should be given by the commutator of covariant derivatives. The commutators of $\hat{R}_{+i}$ and $\hat{R}_{-i}$ are in the Lie algebra of $U(k)$, so we can specify the background field by specifying the right action of $U(k)$ on the wave functions. For the constant background field, the relevant conditions are

$$
\begin{gathered}
\hat{R}_{a} \Psi_{m ; \alpha}^{J}(g)=\left(T_{a}\right)_{\alpha \beta} \Psi_{m ; \beta}^{J}(g) \\
\hat{R}_{k^{2}+2 k} \Psi_{m ; \alpha}^{J}(g)=-\frac{n k}{\sqrt{2 k(k+1)}} \Psi_{m ; \alpha}^{J}(g)
\end{gathered}
$$

where $m(=1, \ldots, \operatorname{dim} J)$ counts the degeneracy of the Landau level. Equation (45) shows that the wave functions $\Psi_{m ; \alpha}^{J}$ transform, under right rotations, as a representation $\tilde{J}$ of $S U(k)$. Here $\left(T_{a}\right)_{\alpha \beta}$ are the representation matrices for the generators of $S U(k)$ in the representation $\tilde{J}$, and $n$ is an integer characterizing the Abelian part of the background field. $\alpha, \beta$ label states within the $S U(k)$ representation $\tilde{J}$ (which is itself contained in the representation $J$ of $S U(k+1))$. The index $\alpha$ carried by the wave functions $\Psi_{m ; \alpha}^{J}(g)$ is basically the gauge index. The wave functions are sections of a $U(k)$-bundle on $\mathbb{C} \mathbb{P}^{k}$. In terms of $\mathcal{D}$-functions, they are given by $\Psi_{m ; \alpha}^{J}(g)=\sqrt{\operatorname{dim} J}\langle J, m|g| J, \alpha, n\rangle$.

The Hamiltonian $H$ for the Landau problem is proportional to the covariant Laplacian on $\mathbb{C P}^{k}$; explicitly the action of $H$ on wave functions is given by

$$
H \Psi=-\frac{1}{4 m}\left(\mathcal{D}_{+i} \mathcal{D}_{-i}+\mathcal{D}_{-i} \mathcal{D}_{+i}\right) \Psi
$$

Since the commutator of $\left[\hat{R}_{+i}, \hat{R}_{-i}\right]$ is in the algebra of $U(k)$, we see from (44) and (46) that $H$ is proportional to $\sum_{i} \hat{R}_{+i} \hat{R}_{-i}$, apart from additive constants. Thus the lowest Landau level should satisfy, in addition to the requirements (45), (46), the condition

$$
\hat{R}_{-i} \Psi=0
$$

This is the holomorphicity condition on the lowest Landau level wave functions.

We consider, for simplicity, the case of a $U(1)$ background, taking $|J, \mathfrak{r}\rangle$ to correspond to the trivial (singlet) representation for $S U(k) \in S U(k+1)$. The relevant representations are then the rank $n$ totally symmetric representations of $S U(k+1)$ and we can construct them explicitly using complex coordinates for $\mathbb{C} \mathbb{P}^{k}$ as

$\Psi_{i_{1} i_{2} \cdots i_{k}}=\sqrt{N}\left[\frac{n !}{i_{1} ! i_{2} ! \cdots i_{k} !(n-s) !}\right]^{\frac{1}{2}} \frac{z_{1}^{i_{1}} z_{2}^{i_{2}} \cdots z_{k}^{i_{k}}}{(1+\bar{z} \cdot z)^{\frac{n}{2}}}$

where $s=i_{1}+i_{2}+\cdots+i_{k}$ and $N=\operatorname{dim} J=(n+k) ! /(n ! k !)$ is the total number of states or degeneracy of the LLL. The volume element for $\mathbb{C P}^{k}$ is

$$
d \mu=\frac{k !}{\pi^{k}} \frac{d^{2} z_{1} \cdots d^{2} z_{k}}{(1+\bar{z} \cdot z)^{k+1}}
$$

We have chosen the normalization such that the total volume, $\int d \mu$, is 1 . For the entanglement entropy, we thus need

$$
\begin{aligned}
\lambda_{i_{1} i_{2} \cdots i_{k}} & =\int_{0}^{R} d \mu \Psi_{i_{1} i_{2} \cdots i_{k}}^{*} \Psi_{i_{1} i_{2} \cdots i_{k}} \\
& =\frac{(n+k) !}{(s+k-1) !(n-s) !} \int_{0}^{R^{2}} d u \frac{u^{s+k-1}}{(1+u)^{n+k+1}}
\end{aligned}
$$

where, in the second line, we have carried out the angular integrations taking the interface to be spherically symmetric. The maximal difference of $\lambda_{s}$ for nearby values of $s$ is now obtained for $s=s_{*}=w(n+k-1)-(k-1)$, and 


$$
\begin{aligned}
\frac{\lambda_{s_{*}}-\lambda_{s_{*}+1}}{\lambda_{s_{*}}} & =\sqrt{\frac{2}{w(1-w) \pi}} \frac{1}{\sqrt{n+k-1}}+\cdots, \\
w & =\frac{R^{2}}{1+R^{2}} .
\end{aligned}
$$

As before, we then find that all values between zero and 1 are realized for some $\lambda_{s}$, leading to the same conclusion:

For all allowable states in the framework of using the lowest Landau as a model for a noncommutative version of $\mathbb{C P}^{k}, \cap_{\Psi} \operatorname{Spec}\left(\Delta_{\Psi}\right)=\mathbb{R}_{+}$.

We have shown this result only for the case of a $U(1)$ background. We expect this to hold even with additional non-Abelian background fields. This will be taken up in a subsequent paper.

\section{ARBITRARY BACKGROUND FIELDS AND SPIN CONNECTION}

We now turn to the second issue mentioned in the Introduction, namely, the dependence of the entanglement entropy on the background fields and the spin connection. For this, we need to know how $u_{s}^{*} u_{s}$ depends on these quantities. One way to identify this dependence is to write the Hamiltonian (47) where the covariant derivatives include additional background fields and then use perturbation theory to calculate the change in $u_{s}^{*} u_{s}$. A limit of $m \rightarrow 0$ may then be taken at the end to isolate the LLL wave functions. A simpler alternative, which we shall consider here, is to utilize previous calculations for the effective action for droplets of fermions [11]. The basic strategy is the following. We will consider a general occupancy matrix for a subset of the states of the lowest Landau level. We can then define a function similar to the symbol for this matrix which carries information about the wave functions. A general ansatz for this function can then be written down. Using an index theorem appropriate to the states of the LLL and considering special cases we can firm up the various terms in the ansatz. This will then yield the leading terms for the background dependence of $u_{s}^{*} u_{s}$. This part of the reasoning will rely on [19] where the Dolbeault index density was used to obtain the bulk effective action for the $\nu=1$ state.

Before proceeding to the main line of reasoning, we assemble two key ingredients, namely, the index theorem and the generalized Chern-Simons form. The wave functions of the lowest Landau level obey a holomorphicity condition, which is (48) for $\mathbb{C P}^{k}$ and a suitable generalization of the same for other complex manifolds. The background fields are included in the relevant antiholomorphic derivatives via conditions like (45), (46). Thus we are looking for the kernel of the antiholomorphic covariant derivatives on $\mathcal{M}$. This is given by the Dolbeault index, with the index density

$$
\begin{aligned}
\mathcal{I}_{\text {Dolb }}= & \operatorname{td}\left(T_{c} \mathcal{M}\right) \wedge \operatorname{ch}(V) \\
= & \frac{1}{2 \pi} \operatorname{Tr}\left(F+\frac{1}{2} R\right) \\
& -\frac{1}{8 \pi^{2}}\left[\operatorname{Tr} F^{2}+\operatorname{Tr} R \operatorname{Tr} F+\frac{1}{4}(\operatorname{Tr} R)^{2}-\frac{1}{12} \operatorname{Tr} R^{2}\right]+\cdots
\end{aligned}
$$

where $F$ is the two-form field strength for the gauge field and $R$ is the curvature two-form. In this equation $\operatorname{td}\left(T_{c} \mathcal{M}\right)$ is the Todd class on the complex tangent space of the manifold $\mathcal{M}$. Rather than give the general formula using a splitting principle, we display the expansion in powers of the curvature. The general formula is given in [20] and is discussed in the specific context of Landau level states in [19]. Also, in (53) $\operatorname{ch}(V)=\operatorname{Tr}\left(e^{i F / 2 \pi}\right)$ is the Chern character of the vector bundle $V$. (The charged fields defining the Landau problem are sections of this vector bundle; i.e., they have $\operatorname{dim} V$ components corresponding to the representation for the non-Abelian gauge group, each component being a local function on $\mathcal{M}$. For example, for $\mathbb{C P}^{k}$, we can consider fields $\phi_{\alpha}$ with wave functions of the form $\Psi_{m ; \alpha}^{J}(g)=\sqrt{\operatorname{dim} J}\langle J, m|g| J, \alpha, n\rangle$ as in Sec. IV. In this case, $\operatorname{dim} V=\operatorname{dim} \tilde{J}$ which is the dimension of the $U(k)$ representation carried by the state $|J, \alpha, n\rangle$. For more details, see [19].) As with such formulas, for twodimensional manifolds we use the two-form part of $\mathcal{I}_{\text {Dolb }}$ from (53), for four-manifolds we use the four-form part, etc.

Turning to the second ingredient, namely, the generalized Chern-Simons form, notice that the index densities involve symmetrized traces of powers of the gauge field strength (as a two-form), or powers of the curvature twoform, or mixed terms involving products of powers of both. Now, if $\mathcal{P}(F)$ is an invariant polynomial which is the symmetrized trace of a product of $k F^{\prime}$ s, then

$$
\begin{aligned}
\mathcal{P}\left(F^{(1)}\right)-\mathcal{P}\left(F^{(2)}\right) & =d Q\left(A^{(1)}, A^{(2)}\right) \\
Q\left(A^{(1)}, A^{(2)}\right) & =k \int_{0}^{1} d t \mathcal{P}\left(A^{(1)}-A^{(2)}, F_{t}, F_{t}, \ldots, F_{t}\right),
\end{aligned}
$$

where $A_{t}=A^{(2)}+t\left(A^{(1)}-A^{(2)}\right)$, with $F_{t}=d A_{t}+A_{t}^{2}$. Notice that $A_{t}$ defines a straight line in the space of potentials connecting the two potentials $A^{(2)}$ and $A^{(1)}$. Equation (54) is the definition of the generalized ChernSimons term $Q\left(A^{(1)}, A^{(2)}\right)$ [20]. This version of the ChernSimons form has been used in physics contexts before, for example, in obtaining expressions for gauge (and gravitational) anomalies with a nontrivial gauge (or gravitational) background [21]. We may also note that, since $\mathcal{P}(F)$ can be written as the derivative of a Chern-Simons term C.S. $(A)$, (54) is equivalent to writing 
$Q\left(A^{(1)}, A^{(2)}\right)=$ C.S. $\left(A^{(1)}\right)-$ C.S. $\left(A^{(2)}\right)+d B\left(A^{(1)}, A^{(2)}\right)$.

The formula (54) has the advantage of providing an explicit expression for $B\left(A^{(1)}, A^{(2)}\right)$ as well. Even though we used the notation of $A$ and $F$ in Eqs. (54) and (55), the statements equally well apply to the spin connection and the curvature.

We now consider the case of $M$ of the lowest Landau levels being occupied. (Eventually, we will be interested in the $\nu=1$ state with all states being occupied. What we outline here with a smaller droplet is only a trick to get the background dependence of $u_{s}^{*} u_{s}$.) We can specify the droplet of $M$ occupied states by the occupancy matrix $P$ which is given by

$$
P_{i j}= \begin{cases}\delta_{i j}, & i, j=0,1, \ldots,(M-1) \\ 0 & i, j \geq M .\end{cases}
$$

Corresponding to this occupancy matrix, we introduce the function $^{4}$

$$
(P)_{M-1}=\sum_{0}^{M-1} u_{s}^{*} u_{s}
$$

Thus we may write $u_{M}^{*} u_{M}=(P)_{M}-(P)_{M-1}$. The function $(P)$ is basically the number density of particles in the lowest Landau level, and hence it should be proportional to the index density for the $\nu=1$ state, modulo terms which integrate to zero. This is the point of utility of the index theorem.

The background field dependence of $u_{s}^{*} u_{s}$ involves the comparison of two choices of the background. We will denote the background fields we start with by $a$ and $\omega^{(0)}$, where $a$ is the potential for the gauge part and $\omega^{(0)}$ is the spin connection. For example, for $\mathbb{C} \mathbb{P}^{k}$, the Abelian part of $a$ will be an appropriate multiple of the Kähler form; there can be a non-Abelian background as well. The spin connection $\omega^{(0)}$ will correspond to the standard curvatures for $\mathbb{C P}^{k}$ with the Fubini-Study metric. The general background we want to consider will have gauge fields $a+A$ and spin connection $\omega$. We are thus interested in the function $(P)$ calculated with the one-particle wave functions corresponding to the background $\left(a, \omega^{(0)}\right)$ and with those corresponding to the background $(a+A, \omega)$. We can then use the result $u_{M}^{*} u_{M}=(P)_{M}-(P)_{M-1}$ to identify the background dependence.

An important point is that the new values of the background fields, i.e., $(a+A, \omega)$, must be such that the total

\footnotetext{
${ }^{4}$ This is related to what is called the symbol for $P$ by a factor of $N$, the total degeneracy of the LLL. The symbol is defined using just the group elements, say $\langle J, m|g| J, \alpha, n\rangle$ for $\mathbb{C P}^{k}$. Thus the symbol of $P$ is $\frac{1}{N}(P)[10,11]$.
}

number of states obtained by quantization remains the same. Thus the fields we are considering must all be in the same topological class, so that the Dolbeault index is unaltered by $a \rightarrow a+A, \omega^{(0)} \rightarrow \omega$. In this sense, we may think of the new fields as a perturbation of the old ones.

We can now write down a general ansatz for $(P)$ as

$$
(\tilde{P})=\frac{\mathcal{I}_{\mathrm{Dolb}}}{N}(P)^{(0)}-K d(P)^{(0)}+d \mathbb{X}
$$

Here $(P)^{(0)}$ denotes the function corresponding to $P$ calculated with the unperturbed one-particle wave functions, namely, with the background $A^{(2)}=\left(a, \omega^{(0)}\right)$. On the left-hand side, we have the function for $P$ calculated with the perturbed wave functions corresponding to $A^{(1)}=(a+A, \omega)$. We actually use the dual on the lefthand side so that it can be viewed as a $2 k$-form; this is signified by the tilde sign. $(\tilde{P})$ is to be viewed as the function $(P)$ multiplied by the volume form appropriate to the background $(a+A, \omega)$. Further, $K$ is a $(2 k-1)$-form, so is $\mathbb{X}$. The nature of the terms in (58) and the justification for them can be seen from the following observations.

(1) First consider the case where all states are filled, so that $M=N$. When all states are filled, $(P)^{(0)}$ is a constant, in fact equal to $N$ for large $n$, as seen from $[11,22]$. (The symbol is equal to 1 , but since we have used the normalized wave functions, $(P)^{(0)}=N$ in this case.) We see that (58) implies that $(\tilde{P})=\mathcal{I}_{\text {Dolb }}$, provided $d \mathbb{X}$ also involves only derivatives of $(P)^{(0)}$. The result $(\tilde{P})=\mathcal{I}_{\text {Dolb }}$ is as it should be, since $(\tilde{P})$ is the number density of the occupied states and it should be the index density when all states are occupied.

(2) Second, consider the case when the additional background fields are zero, i.e., we have only $\left(a, \omega^{(0)}\right)$, but keeping $M<N$. In this case, we expect $(\tilde{P})=d \mu(P)^{(0)}$. In (58), we can thus set $\mathcal{I}_{\text {Dolb }}=\mathcal{I}_{\text {Dolb }}\left(a, \omega^{(0)}\right)$. Further, we have

$$
\mathcal{I}_{\text {Dolb }}\left(a, \omega^{(0)}\right)=N d \mu .
$$

The factor of $N$ in this formula is easily understood. It is needed to ensure that the integral of $\int \mathcal{I}_{\text {Dolb }}\left(a, \omega^{(0)}\right)$ gives $N$ for the unperturbed case, since we normalized the unperturbed volume element to integrate to 1 . We see that (58) consistently reduces to $(\tilde{P})=d \mu(P)^{(0)}$, provided both $K$ and $\mathbb{X}$ vanish when the additional gauge fields are set to zero.

(3) Continuing with the case of $M<N$, we expand $\mathcal{I}_{\text {Dolb }}$ around $\left(a, \omega^{(0)}\right)$ using (54), i.e.,

$$
\mathcal{I}_{\text {Dolb }}(a+A, \omega)=\mathcal{I}_{\text {Dolb }}\left(a, \omega^{(0)}\right)+d Q
$$


Using this relation, (58) becomes

$$
(\tilde{P})=d \mu(P)^{(0)}+\frac{d Q}{N}(P)^{(0)}-K d(P)^{(0)}+d \mathbb{X}
$$

Since the total number of states should be the same for both $\left(a, \omega^{(0)}\right)$ and $(a+A, \omega)$, terms in (58) other than $d \mu(P)^{(0)}$ must combine into a total derivative, so that they can give zero upon integration. This identifies $K=Q / N$.

(4) Finally, we have already mentioned that $\mathbb{X}$ must vanish when the additional gauge fields are set to zero and that it should also vanish when $(P)^{(0)}$ is a constant, from items 2 and 1 above. Therefore $\mathbb{X}$ can be written as $W^{i} \partial_{i}(P)^{(0)}$, where $W^{i}$ is a $(2 k-1)$ form and is also a vector. It is not determined by our arguments so far.

Collecting all these results together, we can now rewrite (58) as

$$
(\tilde{P})=d \mu(P)^{(0)}+d\left[\frac{Q P^{(0)}}{N}\right]+d\left(W^{i} \partial_{i}(P)^{(0)}\right)
$$

where $W^{i}$ is zero when restricted to $a, \omega^{(0)}$. As mentioned before, the relevant index density we should use for the generalized Chern-Simons form $Q$ will be the Dolbeault index density $\mathcal{I}_{\text {Dolb }}$, with $A^{(1)}=(a+A, \omega)$ and $A^{(2)}=\left(a, \omega^{(0)}\right)$.

Taking the difference between $(P)_{M}$ and $(P)_{M-1}$, we conclude that

$d \mu\left[u_{s}^{*} u_{s}\right]_{a+A, \omega}=d \mu u_{s}^{*} u_{s}+d\left[\frac{Q u_{s}^{*} u_{s}}{N}\right]+d\left(W^{i} \partial_{i}\left(u_{s}^{*} u_{s}\right)\right)$

$u_{s}^{*} u_{s}$ in all terms on the right-hand side is evaluated with the unperturbed background $\left(a, \omega^{(0)}\right)$. Going back to the fully filled states, i.e., $\nu=1$, we can now integrate (63) over a region $D$ whose boundary corresponds to the entangling surface. The result is

$$
\begin{aligned}
{\left[\lambda_{s}\right]_{a+A, \omega}=} & {\left[\lambda_{s}\right]_{a, \omega^{(0)}}+\frac{1}{N} \oint_{\partial D} Q\left[u_{s}^{*} u_{s}\right]_{a, \omega^{(0)}} } \\
& +\oint_{\partial D} W^{i}\left[\partial_{i}\left(u_{s}^{*} u_{s}\right)\right]_{a, \omega^{(0)}}
\end{aligned}
$$

The $\lambda_{s}$-terms in this equation is the result after integrating over the boundary $\partial D$; i.e., the analogue of the angular integrations has been carried out as discussed earlier and in the Appendices.

We can now use (64) for the entropy. In the spirit of perturbation theory, the change in the entanglement entropy due to the change in the background fields is given by

$$
\begin{aligned}
S= & S\left(a, \omega^{(0)}\right)-\sum_{s} \log \left(\frac{\lambda_{s}}{1-\lambda_{s}}\right) \delta \lambda_{s} \\
= & S\left(a, \omega^{(0)}\right)-\oint_{\partial D} \frac{Q}{N} \sum_{s} \log \left(\frac{\lambda_{s}}{1-\lambda_{s}}\right)\left[u_{s}^{*} u_{S}\right]_{\partial D ; a, \omega^{(0)}} \\
& -\sum_{s} \log \left(\frac{\lambda_{s}}{1-\lambda_{s}}\right) \oint_{\partial D} W^{i}\left[\partial_{i}\left(u_{s}^{*} u_{s}\right)\right]_{\partial D ; a, \omega^{(0)}}
\end{aligned}
$$

This is the main result of this section, summarizing our expectation for the background dependence in terms of $Q$. The precise form of the remaining factors is not important regarding the background dependence. The arguments which led to this result are very general, but indirect, based on index theorems. We will carry out an explicit calculation, which is presented in Appendix B, for some special cases. (We expect to present the explicit calculations for the more general cases in a separate paper.) This will show that the second correction from (64), namely, $W^{i}\left[\partial_{i}\left(u_{s}^{*} u_{s}\right)\right]_{a, \omega^{(0)}}$ may be taken to be subdominant compared to the first, in some qualified sense. Taking this into account, we may restate the result as follows.

The leading term in the dependence of the entanglement entropy on the gauge fields and spin connection is proportional to the generalized Chern-Simons term $Q$ for the Dolbeault index density.

It may be useful at this stage to see the explicit formulas for the generalized Chern-Simons forms relevant to some lower dimensional examples, rather than the more cryptic expression (54). Using (53) and (54) we find

$$
Q_{2 d}=\frac{1}{2 \pi} \operatorname{Tr}\left(A+\frac{1}{2}\left(\omega-\omega^{(0)}\right)\right)
$$

$$
\begin{aligned}
Q_{4 d}= & -\frac{1}{8 \pi^{2}}[-4 \pi \text { C.S. }(a+A)+4 \pi \text { C.S. }(a)+d \operatorname{Tr}(a A) \\
& -\frac{1}{12}\left(-4 \pi \text { C.S. }(\omega)+4 \pi \text { C.S. }\left(\omega^{(0)}\right)+d \operatorname{Tr}\left(\omega^{(0)}\left(\omega-\omega^{(0)}\right)\right)\right) \\
& +\operatorname{Tr}\left(\omega-\omega^{(0)}\right) \operatorname{Tr}(d A)+\operatorname{Tr}\left(\omega-\omega^{(0)}\right) \operatorname{Tr}\left(d a+a^{2}\right)+\operatorname{Tr}(A) \operatorname{Tr}\left(d \omega^{(0)}\right) \\
& \left.+\frac{1}{2} \operatorname{Tr}\left(\omega-\omega^{(0)}\right) \operatorname{Tr}\left(d \omega^{(0)}\right)+\frac{1}{4} \operatorname{Tr}\left(\omega-\omega^{(0)}\right) \operatorname{Tr}\left(d \omega-d \omega^{(0)}\right)\right] .
\end{aligned}
$$


Here C.S. stands for the Chern-Simons three-form, given, for a generic argument $A$, as

$$
\text { C.S. }(A)=-\frac{1}{4 \pi} \operatorname{Tr}\left(A d A+\frac{2}{3} A^{3}\right) .
$$

\section{DISCUSSION}

The main results of this paper are about the spectrum of the modular operator and the dependence of the entanglement entropy on the background fields and spin connection, for a noncommutative space, or equivalently, for the $\nu=1$ quantum Hall state. These have been spelled out at the end of Secs. III and V. An important direction to explore further is the term involving $\left(W^{i} \partial_{i}\left(u_{s}^{*} u_{s}\right)\right)$ in (62), (63). This is presumably related to the boundary actions for a droplet of finite size. In the context of quantum Hall effect, while any direct experimental implication is unclear, our result is in the nature of elucidating general properties of Hall states.

As for the context of noncommutative geometry, the following comments may help with the placement of our results in a larger context. In the Introduction we have already alluded to the nexus of ideas about entropy and gravity, entanglement as an integral feature of relativistic field theory, and noncommutative geometry which attributes degrees of freedom to space itself. To this we may add the observation that, in $2+1$ dimensions, standard Einstein gravity can be described by an action which is the difference of two Chern-Simons terms [12]. In higher dimensions, one can consider a class of gravity theories with Chern-Simons actions, although they do not correspond to the standard Einstein gravity [13]. In all these cases, the field equations of gravity arise as extremization of an action which is a combination of Chern-Simons terms. Our result that the leading term in the background dependence of the entanglement entropy in noncommutative geometry (modeled as quantum Hall systems) is given by a generalized Chern-Simons term takes on added significance when viewed within this circle of ideas. We expect that this result can be utilized to develop an approach to gravity in odd dimensional spacetimes based on noncommutative geometry by modeling space by quantum Hall systems and that such a description would naturally realize the field equations for gravity as maximization conditions for the entanglement entropy. We plan to explore this idea further in future publications.

\section{ACKNOWLEDGMENTS}

I thank Dimitra Karabali and Alexios Polychronakos for reading the manuscript carefully and providing many useful comments which have significantly improved the presentation. I also thank A. Polychronakos for pointing out a missing term in an earlier derivation of the formula (52). I am also grateful to A. Abanov for comments and to
A. Abanov and P. Ghaemi for some of the relevant references. This research was supported in part by the U.S. National Science Foundation Grant No. PHY1820721 and by Professional Staff Congress-City University of New York awards.

\section{APPENDIX A: ASYMPTOTIC FORMULAS FOR $\lambda_{s}$}

We will start with Eq. (51) from the text, which gives

$$
\lambda_{s}=\frac{(n+k) !}{(s+k-1) !(n-s) !} \int_{0}^{R^{2}} d u \frac{u^{s+k-1}}{(1+u)^{n+k+1}}
$$

This formula applies to $\mathbb{C P}$. We started in the text with the case of $k=1$ for $\mathbb{C P} \mathbb{P}^{1} \sim S^{2}$ and with $R=1$. Those results can be obtained as special cases of the formulas give here.

We first consider small values of $s$ compared to $n$. The integrand in (A1) is a function which peaks around some value of $u$. This value is near zero for small $s$, moving to large values of $u$ as $s$ becomes large. Thus, for small values of $s$ compared to $n, \lambda_{s}$ will be close to 1 . The full integral, up to infinity, gives 1 , so we can rewrite (A1) as

$$
\lambda_{s}=1-\frac{(n+k) !}{(s+k-1) !(n-s) !} \int_{R^{2}}^{\infty} d u \frac{u^{s+k-1}}{(1+u)^{n+k+1}}
$$

Making a change of variables $u=x /(n+k+1)$, we find

$$
\begin{aligned}
\lambda_{s}= & 1-\frac{(n+k) !}{\Gamma(s+k)(n-s) !} \frac{1}{(n+k+1)^{s+k}} \\
& \times \int_{R^{2}(n+k+1)}^{\infty} d x x^{s+k-1} e^{-x}\left(1+\frac{x^{2}}{2(n+k+1)}+\cdots\right) \\
\approx & 1-\frac{\Gamma\left(s+k, R^{2}(n+k+1)\right)}{\Gamma(s+k)}+\cdots \\
\approx & 1-\frac{\left[R^{2}(n+k+1)\right]^{s+k-1}}{\Gamma(s+k)} e^{-R^{2}(n+k+1)}+\cdots
\end{aligned}
$$

where, in the first line, we used

$$
\left(1+\frac{x}{N}\right)^{N} \approx e^{x}\left(1-\frac{x^{2}}{2 N}+\cdots\right), \quad \text { as } N \rightarrow \infty .
$$

For the second and third lines we used the definition of the incomplete $\Gamma$-function and its asymptotic expansion [23],

$$
\begin{aligned}
\Gamma(s+k, X) & =\int_{X}^{\infty} d x x^{s+k-1} e^{-x} \\
& \approx X^{s+k-1} e^{-X}+\cdots
\end{aligned}
$$

Equation (A3) shows that $\lambda_{s}$ is exponentially close to 1 for small values of $s$, as $n$ becomes large. The ellipsis indicates terms which are smaller than what is displayed.

For values of $s$ close to $n$, we can do a similar analysis. Writing $s=n-r$ and carrying out an inversion $u=(n+k+1) / x$, we find 


$$
\begin{aligned}
\lambda_{n-r}= & \frac{(n+k) !}{\Gamma(n-r+k) r !} \frac{1}{(n+k+1)^{r+1}} \\
& \times \int_{\frac{(n+k+1)}{R^{2}}}^{\infty} d x x^{r} e^{-x}\left(1+\frac{w^{2}}{2(n+k+1)}+\cdots\right) \\
\approx & \frac{1}{\Gamma(r+1)}\left(\frac{n+k+1}{R^{2}}\right)^{r} e^{-(n+k+1) / R^{2}}+\cdots
\end{aligned}
$$

We see that, for $s$ near $n$, the values are exponentially small.

The maximal difference between nearby values of $s$ will occur near the midpoint of the transition from 1 to zero. For this, $s$ is also large and one can use a semiclassical or steepest descents method. Making a change of variable to $t=u /(1+u)$, (A1) can be written as an incomplete beta function,

$$
\begin{aligned}
\lambda_{s} & =\frac{(n+k) !}{\Gamma(s+k)(n-s) !} \int_{0}^{w} d t t^{s+k-1}(1-t)^{n-s} \\
& =\frac{(n+k) !}{\Gamma(s+k)(n-s) !} \int_{0}^{w} d t e^{F(t)} \\
F(t) & =(s+k-1) \log t+(n-s) \log (1-t)
\end{aligned}
$$

where $w=R^{2} /\left(1+R^{2}\right)$. The minimum of $F(t)$ occurs at $t_{0}=(s+k-1) /(n+k-1)$. Expanding $F(t)$ around this value, $E^{F(t)}$ becomes a Gaussian function centered around this value of $t$. If the maximum of the Gaussian is well within the range of integration, which is the case for small $s$, we will find $\lambda_{s} \approx 1$. If the center of the Gaussian is well beyond the range of integration, which is the case for $s$ near $n$, we will find $\lambda_{s} \approx 0$. The midpoint of the transition occurs for $t_{0}$ at the upper limit of integration, namely, for $t_{0}=w$, which corresponds to $s=s_{*}$ given by

$$
\begin{aligned}
s_{*} & =w(n+k-1)-(k-1), \\
n-s_{*} & =(1-w)(n+k-1)
\end{aligned}
$$

Expanding $F(t)$ around $t_{0}$, we obtain

$$
\begin{aligned}
F(t)= & (n+k-1)\left[\left[t_{0} \log t_{0}+\left(1-t_{0}\right) \log \left(1-t_{0}\right)\right]\right. \\
& \left.-\frac{x^{2}}{2 t_{0}\left(1-t_{0}\right)}+\frac{\left(1-2 t_{0}\right) x^{3}}{3 t_{0}^{2}\left(1-t_{0}\right)^{2}}+\cdots\right]
\end{aligned}
$$

where $x=t-t_{0}$. Using this expression, we find

$$
\lambda_{s_{*}} \approx \frac{(n+k) !}{\Gamma\left(s_{*}+k\right) \Gamma\left(n-s_{*}+1\right)} e^{F\left(t_{0}\right)}\left[\sqrt{\frac{\pi t_{0}\left(1-t_{0}\right)}{2(n+k-1)}}-\frac{2\left(1-2 t_{0}\right)}{3(n+k-1)}+\cdots\right]
$$

where $t_{0}=w$. It should be noted that the range of integration for $x$ for this case is from $-\infty$ to zero.

We do a similar calculation for $\lambda_{s_{*}+1}$. For $s=s_{*}+1$, the minimum of $F(t)$ occurs at

$$
t_{1}=\frac{s_{*}+k}{n+k-1}=w+\epsilon, \quad \epsilon=\frac{1}{n+k-1}
$$

This is just beyond the range of integration. The integral over $x$ now becomes

$$
\int_{0}^{w} d t e^{F(t)} \approx e^{F\left(t_{1}\right)} \int_{-\infty}^{-\epsilon} d x \exp \left(-\frac{(n+k-1) x^{2}}{2 t_{1}\left(1-t_{1}\right)}+\frac{(n+k-1)\left(1-2 t_{1}\right) x^{3}}{3 t_{1}^{2}\left(1-t_{1}\right)^{2}}+\cdots\right) .
$$

The integral with the upper limit as zero is similar to what was encountered for $\lambda_{s_{*}}$, but we have to subtract out the integral from $-\epsilon$ to zero. Apart from this, the result is of the form in (A10) with $s_{*} \rightarrow s_{*}+1$ and with $t_{0} \rightarrow t_{1}=w+\epsilon$. The final result is thus

$$
\begin{aligned}
\lambda_{s_{*}+1} \approx & \frac{(n+k) !}{\Gamma\left(s_{*}+k+1\right) \Gamma\left(n-s_{*}\right)} e^{F(w+\epsilon)}\left[\sqrt{\frac{\pi(w+\epsilon)(1-w-\epsilon)}{2(n+k-1)}}-\frac{2(1-2 w-2 \epsilon)}{3(n+k-1)}+\cdots\right] \\
& -\epsilon \frac{(n+k) !}{\Gamma\left(s_{*}+k+1\right) \Gamma\left(n-s_{*}\right)} e^{F(w+\epsilon)}
\end{aligned}
$$

The rest of the simplification is straightforward, using properties of the $\Gamma$-functions. The leading term in the difference $\lambda_{s_{*}}-\lambda_{s_{*}+1}$ comes from the second line of (A13). This leads to the expression (52) quoted in text,

$$
\frac{\lambda_{s_{*}}-\lambda_{s_{*}+1}}{\lambda_{s_{*}}}=\sqrt{\frac{2}{w(1-w) \pi}} \frac{1}{\sqrt{n+k-1}}+\cdots
$$




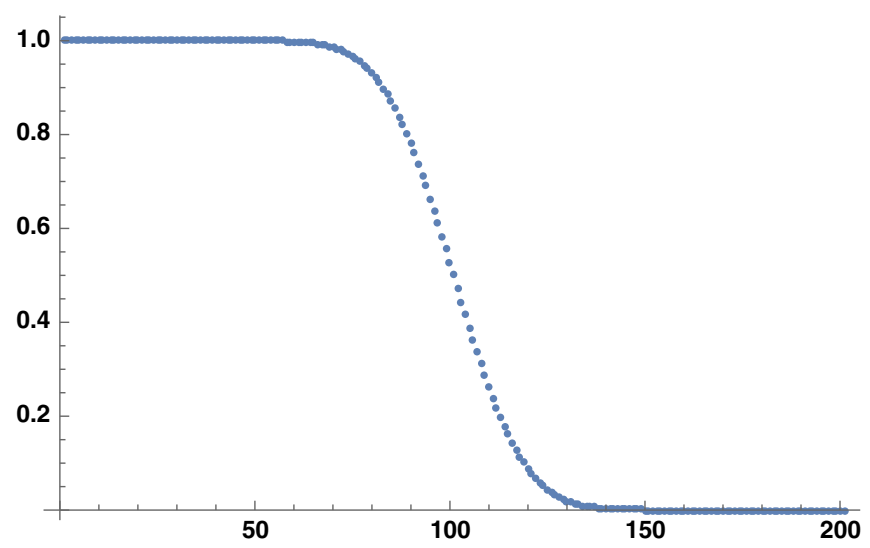

FIG. 1. Values of $\lambda_{s}$ as a function of $s$, for $n=200$.

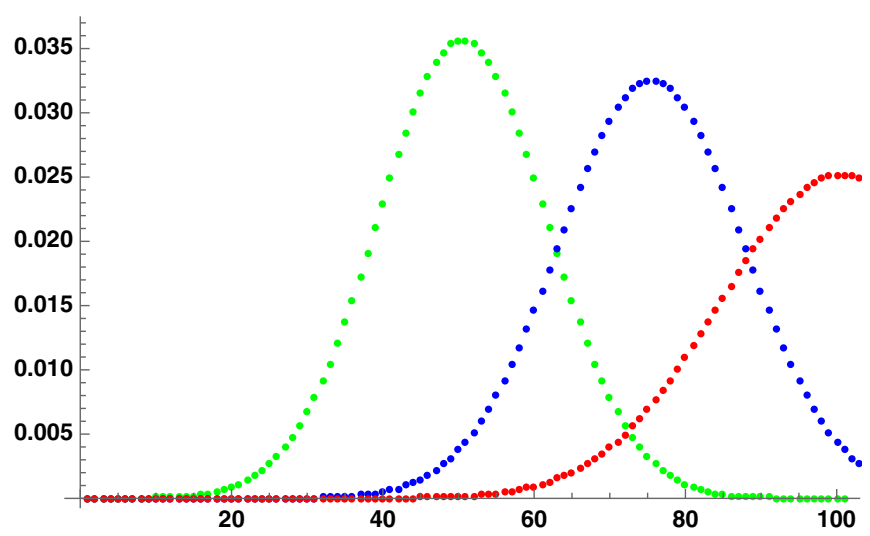

FIG. 2. Values of $\lambda_{s}-\lambda_{s+1}$ for $n=100,150,200$, for the three curves from left to right.

The results obtained in this Appendix can also be checked numerically. As an example, consider the case of the two-sphere or $k=1$. In this case, taking $R=1$ (i.e., $w=\frac{1}{2}$ ), we have plotted, in Fig. 1, we have plotted the values of $\lambda_{s}$ for $n=200$; the result shows that the values are close to zero and 1 at the two ends and has the largest separation between $\lambda_{s}$ and $\lambda_{s+1}$ for $s_{*}=n / 2$. The second graph (Fig. 2) shows the differences $\left(\lambda_{s_{*}}-\lambda_{s_{*}+1}\right)$. We see that the maximum values of the differences decrease as we increase $n$. The peak value can be checked to be as given by (A14) or (52).

\section{APPENDIX B: DIRECT CALCULATION OF BACKGROUND FIELD DEPENDENCE}

In this Appendix, we will go over the explicit calculation of some of the terms which arise in the dependence of $u_{s}^{*} u_{s}$ on the background fields. In other words, we go over the calculation of the terms in (63), (64). The basic strategy is to consider $\operatorname{Tr}\left(P A_{0}\right)$, rather than just the function (57) for $P$, since this can be related to the calculation of the effective action. For simplicity, we will consider only the changes in the background gauge fields, keeping the spin connection as $\omega^{(0)} \cdot \operatorname{Tr}\left(P A_{0}\right)$ can be expressed as an integral over the phase space with the star product of the functions corresponding to $P$ and $A_{0}$ as the integrand. But instead of considering $P$ and $A_{0}$ as defined by the modified wave functions, we can use the wave functions for the background $\left(a, \omega^{(0)}\right)$, but use $\mathcal{A}$ which is a function of $A_{0}$ and $A_{i}$. In other words,

$$
\begin{aligned}
\operatorname{Tr}\left(P A_{0}\right)= & \int(\tilde{P}) *\left(A_{0}\right) \\
= & \int d \mu \sum_{i j}\left[u_{i}^{*} P_{i j} u_{j}\right]_{a, \omega^{(0)}} * \mathcal{A} \\
= & \int d \mu(P)^{(0)} \mathcal{A} \\
& + \text { terms with derivatives of }(P)^{(0)}, \mathcal{A} .
\end{aligned}
$$

This shows that if we can identify $\mathcal{A}$, then from the first and third lines of this equation, we see that we can obtain the relation between $(\tilde{P})$ and $(P)^{(0)}$, by functional differentiation with respect to $A_{0}$. The calculation of $\mathcal{A}$ has been done in a few different ways; we will go over two methods.

The first method is essentially classical, and can be applied to the case when the background field is Abelian [24]. Let $\Omega$ denote the symplectic structure of the phase space, say, $\mathbb{C P}^{k} ; \Omega$ is a multiple of the Kähler form. The symplectic potential is the Abelian background gauge potential $a$, so that $\Omega=d a$. Changing the gauge field is equivalent to using $\Omega+F=d(a+A)$ as the symplectic two-form. We are interested in $\operatorname{Tr}\left(P A_{0}\right)$ calculated using wave functions with the background $a+A$. The classical version of this is the integral of $A_{0}$ over the phase volume corresponding to $\Omega+F$. So we can write the equivalent of (B1) as

$$
\begin{aligned}
\int d \mu_{\Omega+F} A_{0} & =\int d \mu(P)^{(0)} * \mathcal{A} \\
& =\int d \mu(P)^{(0)} \mathcal{A}+\text { derivative terms }
\end{aligned}
$$

The two-forms $\Omega$ and $\Omega+F$ must belong to the same topological class, so that, upon quantization, we get the same number of states for the Hilbert space. This means that we can use a diffeomorphism to map $\Omega+F$ to $\Omega$. We can then identify $\mathcal{A}$ as the image of $A_{0}$ under this map. More explicitly, there is a diffeomorphism changing the local coordinates $v$ as $v \rightarrow v-w$ such that

$$
\left.\left.\Omega+F]_{v-w}=\Omega\right]_{v}, \quad \mathcal{A}=A_{0}\right]_{v-w}
$$

Equivalently, we can write $\left.a+A]_{v-w}-a\right]_{v}=d f$ for some function $f$. Taking $A$ to be a first order correction, we can solve this equation for $w$ as a series. To the quadratic order, the equations for $w$ are 


$$
\begin{array}{r}
w_{1}^{j} \partial_{j} a_{i}+a_{j} \partial_{i} w_{1}^{j}-A_{i} \approx 0 \\
w_{2}^{j} \partial_{j} a_{i}+a_{j} \partial_{i} w_{2}^{j}+w_{1}^{j} \partial_{j} A_{i}+A_{j} \partial_{i} w_{1}^{j}-\frac{1}{2} w_{1}^{k} w_{1}^{l} \partial_{k} \partial_{l} a_{i}-w_{1}^{k} \partial_{k} a_{j} \partial_{i} w_{1}^{j} \approx 0
\end{array}
$$

where $\approx$ denotes equivalence up to an exact form. The solution to this order is

$$
\begin{aligned}
& w_{1}^{j}=-\left(\Omega^{-1}\right)^{j k} A_{k} \\
& w_{2}^{j}=-\left(\Omega^{-1}\right)^{j k}\left[F_{k l} w_{1}^{l}+\frac{1}{2} w_{1}^{m} w_{1}^{n} \partial_{m} \Omega_{n k}+\frac{1}{2}\left(w_{1}^{m} \partial_{k} w_{1}^{n}\right) \Omega_{m n}\right] .
\end{aligned}
$$

The expression for $\left.\mathcal{A}=A_{0}\right]_{v-w}$ obtained in this manner is

$$
\Omega^{k} \mathcal{A}=\Omega^{k} A_{0}+k \Omega^{k-1} A d A_{0}+\frac{1}{2} k(k-1) \Omega^{k-2} d A A d A_{0}+\cdots+\frac{1}{2} k \Omega^{k-1} d(u \cdot A A)+\cdots
$$

where $u^{i}=\left(\Omega^{-1}\right)^{i j} \partial_{j} A_{0}$. Using this in (B2), we find

$$
\begin{aligned}
\left(u_{s}^{*} u_{s}\right)_{A_{i} \neq 0} & =d \mu\left(u_{s}^{*} u_{s}\right)_{A_{i}=0}+\frac{1}{N} d\left[\left(u_{s}^{*} u_{s}\right)_{A_{i}=0} Q(A, \omega)\right]-\frac{k}{2 N} d\left[\Omega^{k-1} A\left(\Omega^{-1}\right)^{i j} \partial_{i}\left(u_{s}^{*} u_{s}\right)_{A=0} A_{j}\right]+\cdots \\
Q(A) & =k \int_{0}^{1} d t A(\Omega+t d A)^{k-1}
\end{aligned}
$$

There are several observations to be made about this expression. First of all, $\Omega=n \Omega_{K}$ where $\Omega_{K}$ is the Kähler two-form for the space under consideration and $N \approx n^{k} / k$ !. For $\mathbb{C P}^{k}$, we have

$$
\Omega_{K}=i\left[\frac{d z^{i} d \bar{z}^{i}}{(1+\bar{z} \cdot z)}-\frac{\bar{z} \cdot d z z \cdot d \bar{z}}{(1+\bar{z} \cdot z)^{2}}\right] .
$$

Thus terms with $\Omega^{k-1}$ are down by a power of $n$ compared to $\Omega^{k}$-terms. Also, $\left(\Omega^{-1}\right)^{i j}$ gives an additional power of $1 / n$. The series represented by $Q(A, \omega)$ has $(k-1)$ terms terminating with $\Omega^{0}$. Integrating (B8) over the region $D$, we find

$$
\left.\left.\lambda_{s}\right]_{A_{i} \neq 0}-\lambda_{s}\right]_{A_{i}=0}=\frac{Q(A)}{N}\left(u_{s}^{*} u_{s}\right)_{\text {at } R}-\frac{k}{2 N}\left[\Omega^{k-1}\left(\Omega^{-1}\right)^{i j} \partial_{i}\left(u_{s}^{*} u_{s}\right) A_{j}\right]_{\partial D}+\cdots .
$$

Comparing this with (64), we see that we can identify

$W^{i}\left[\partial_{i}\left(u_{s}^{*} u_{s}\right)\right]_{\partial D ; a, \omega^{(0)}}=-\frac{k}{2 N}\left[\Omega^{k-1}\left(\Omega^{-1}\right)^{i j} \partial_{i}\left(u_{s}^{*} u_{s}\right) A_{j}\right]_{\partial D}$

This term is order $A / n^{2}$ while the leading term involving $Q$ is of order $A / n$.

The second method is to consider the time-evolution of the occupancy matrix [25]. This should be a unitary transformation of the form $U(t) P_{0} U^{\dagger}(t)$. The action governing the time-evolution can then be written as

$$
\mathcal{S}=\int d t \operatorname{Tr}\left[P_{0}\left(U^{\dagger} i \frac{\partial U}{\partial t}-U^{\dagger} \mathcal{A} U\right)\right]
$$

It is easy to verify that the variational equation for this is the (quantum) Liouville equation for $P=U(t) P_{0} U^{\dagger}(t)$. The action (B11) can be rewritten using star products as

$$
\mathcal{S}=N \int d \mu d t\left[i\left(P_{0} * U^{\dagger} * \partial_{t} U\right)-\left(P_{0} * U^{\dagger} * \mathcal{A} * U\right)\right]
$$

where $N$ is again the degeneracy. In (B12), all quantities are $c$-number functions, the symbols which use wave functions defined with the background fields $A_{i}$. Rather than working out the perturbed wave functions and symbols directly, we note that the action has the gauge symmetry

$\delta U=-i \theta * U, \quad \delta \mathcal{A}(\vec{x}, t)=\partial_{t} \theta(\vec{x}, t)-i(\theta * \mathcal{A}-\mathcal{A} * \theta)$

for some function $\theta$ on $\mathcal{M}$. The background gauge fields are only defined up to the gauge symmetry

$$
\delta A_{\mu}=\partial_{\mu} \Lambda+i\left[a_{\mu}+A_{\mu}, \Lambda\right], \quad \delta a_{\mu}=0
$$


for some function $\Lambda$ on $\mathcal{M}$. Since (B13) is the only gauge symmetry for the action (B12), the transformation (B14) must induce a transformation of the form (B13). Thus we must have $\mathcal{A}$ and $\theta$ as functions of $a_{\mu}, A_{\mu}, \Lambda$ such that $\mathcal{A}_{\mu}\left(A_{\mu}+\delta A_{\mu}\right) \approx \mathcal{A}+\delta \mathcal{A}$ with $\delta A_{\mu}$ as in (B14) and $\delta \mathcal{A}$ as in (B13). Taking $\mathcal{A}$ to be $A_{0}$ to the lowest order, we can use this idea to solve for $\mathcal{A}$ in terms of the star product (defined in terms of $\Omega$ ). The field $U$ is a boundary field at the edge of the occupied states and can be set to the identity at the end of the calculation. This strategy was used in [25] and leads to

$\mathcal{A}=A_{0}+\frac{1}{4}\left(\Omega^{-1}\right)^{i j}\left\{A_{i}, 2 D_{j} A_{0}+i\left[A_{j}, A_{0}\right]\right\}+\cdots$.

This result is identical to the previous one, if the fields are Abelian.
[1] The recognition of the relation between entropy and gravity goes back to the work of J. Bekenstein and S. Hawking from the 1970s, but for the more modern approach discussing the Einstein equations as a thermodynamic relation, see $\mathrm{T}$. Jacobson, Phys. Rev. Lett. 75, 1260 (1995); 116, 201101 (2016); T. Padmanabhan, Rep. Prog. Phys. 73, 046901 (2010).

[2] There are many papers on entropy in the holographic framework starting with S. Ryu and T. Takayanagi, Phys. Rev. Lett. 96, 181602 (2006); J. High Energy Phys. 08 (2006) 045.

[3] T. Faulkner, M. Guica, T. Hartman, R. C. Myers, and M. V. Raamsdonk, J. High Energy Phys. 03 (2014) 051; B. Swingle and M. van Raamsdonk, arXiv:1405.2933.

[4] E. Verlinde, J. High Energy Phys. 04 (2011) 029.

[5] A. Connes and E. Stormer, J. Funct. Anal. 28, 187 (1978).

[6] R. Clifton and H. Halverson, Stud. Hist. Phil. Mod. Phys. 32, 1 (2001); S. Hollands and K. Sanders, arXiv: 1702.04924; C. J. Fewster and K. Rejzner, arXiv: 1904.04051 .

[7] H. Halverson and M. Mueger, arXiv:math-ph/0602036.

[8] E. Witten, Rev. Mod. Phys. 90, 045003 (2018).

[9] A. Connes, Nocommutative Geometry (Academic Press, New York, 1994); J. Madore, An Introduction to Noncommutative Geometry and its Physical Applications, LMS Lecture Notes 206 (Cambridge University Press, 1995); G. Landi, An Introduction to Noncommutative Spaces and their Geometry, Lecture Notes in Physics, Monographs m51 (Springer-Verlag, Berlin, 1997); For another recent review of fuzzy spaces and theories defined on them, see, A. P. Balachandran, Pramana 59, 359 (2002); A. P. Balachandran and S. Kurkcuoglu, Int. J. Mod. Phys. A 19, 3395 (2004); A. P. Balachandran, S. Kurkcuoglu, and S. Vaidya, arXiv: hep-th/0511114.

[10] For a discussion of noncommutative spaces using the lowest Landau level approach, see D. Karabali and V. P. Nair, J. Phys. A 39, 12735 (2006); D. Karabali, V. P. Nair, and R. Randjbar-Daemi, in From Fields to Strings: Circumnavigating Theoretical Physics, Ian Kogan Memorial Collection, edited by M. Shifman, A. Vainshtein, and J. Wheater (World Scientific, Singapore, 2004), pp. 831-876 and references therein.
[11] D. Karabali and V. P. Nair, Nucl. Phys. B641, 533 (2002); B679, 427 (2004); B697, 513 (2004); For earlier work on Hall effect in higher dimensions, see S. C. Zhang and J. P. Hu, Science 294, 823 (2001); J. P. Hu and S. C. Zhang, Phys. Rev. B 66, 125301 (2002).

[12] A. Achúcarro and P. Townsend, Phys. Lett. B 180, 89 (1986); E. Witten, Nucl. Phys. B311, 46 (1988).

[13] For a recent general review, see J. Zanelli, arXiv:hep-th/ 0502193.

[14] F. D. M. Haldane, Phys. Rev. Lett. 51, 605 (1983). See also Ref. [11]. An early reference on applying geometric quantization for the Landau problem is J. R. Klauder and E. Onofri, Int. J. Mod. Phys. A 04, 3939 (1989).

[15] J. Dubail, N. Read, and E. H. Rezayi, Phys. Rev. B 85, 115321 (2012); 86, 245310 (2012).

[16] I. D. Rodriguez and G. Sierra, Phys. Rev. B 80, 153303 (2009); L. Charles and B. Estienne, Commun. Math. Phys. 376, 521 (2020); B. Estienne and J.-M. Stéphan, Phys. Rev. B 101, 115136 (2020); see also D. Karabali, arXiv: 2006.10920.

[17] H. Li and F. D. Haldane, Phys. Rev. Lett. 101, 010504 (2008); Z. Liu and R. N. Bhatt, Phys. Rev. Lett. 117, 206801 (2016).

[18] For discussions of entanglement entropy for Hall systems from a holographic point of view, see M. Fujita, W. Li, S. Ryu, and T. Takayanagi, J. High Energy Phys. 06 (2009) 066; T. Takayanagi, J. Phys. Conf. Ser. 462, 012053 (2013).

[19] D. Karabali and V. P. Nair, Phys. Rev. D 94, 024022 (2016); 94, 064057 (2016).

[20] See, for example, T. Eguchi, P. B. Gilkey, and A. J. Hanson, Phys. Rep. 66, 213 (1980).

[21] J. Manes, R. Stora, and B. Zumino, Commun. Math. Phys. 102, 157 (1985).

[22] R. Ray and B. Sakita, Ann. Phys. (N.Y.) 230, 131 (1994); Phys. Rev. B 65, 035320 (2001).

[23] See, for example, M. Abramowitz and I. A. Stegun, Handbook of Mathematical Functions (Dover Publications, New York, 1965), p. 263.

[24] V. P. Nair, Nucl. Phys. B750, 289 (2006).

[25] D. Karabali, Nucl. Phys. B726, 407 (2005); B750, 265 (2006). 